\title{
NUMERICAL SIMULATION OF THE EXPLOSTVE EVENTS IN THE SOLAR ATMOSPHERE
}

\author{
Shu-Ping Jiu \\ Department of Earth and Space Science, Iniversity of Science and Technology \\ of C'hina, Hefei, Anhui, People's Republic of China
}

\begin{abstract}
Explosive events are the earliest indicators of flare activity and potentially predict the imminent occurrence of a flare at a specific location. They are highly energetic small-scale phenomena which are frequently detected throughout the quiet and active sun. The observations show that explosive events are related to emerging magnetic flux and t.end to occur on the edges of high photospheric magnentic field regions. The cancellation of photospheric magnetic flux are the manifestation of explosive events, so that they are identified as the magnetic reconnection of flux elements. We assume that emerging flux are convected to the network boundaries with the typical velocity of intranetwork elements. Two-dimension (2D) compressible MHD simulations are performed to explore the reconnection process between emerging intranework flux and network field. The numerical results clearly show the cancellation of magnetic flux and the acceleration of the plasma flow.
\end{abstract}

\section{INTRODUCTION}

The first rocket flight of the Navel Research Laboratory's high resoulution telescopy and spectrograph (HRTS) revealed the widespread occurrence of explosive events in the solar at.mosphere. These events are characterized by small spatial scales ( 2 arc sec), short time scales $(60 \mathrm{~s})$ and high velocity $\left(100 \mathrm{kms}^{-1}\right)$ and are most prominent in spectral lines forned at transition zone temperatures $\left(10^{5} \mathrm{~K}\right)$. It has generally been assumed that the explosive events consist of plasma accelerated by the action of magnetic forces. 'The observatjons show that an unusually high concentration of explosive events is associated with this emerging active region. The positions of the explosive events tend to be located along the neut.ral line separating the opposite magnetic polarities and occur on the edge of the network where the cancellation of magnetic flux would be found. Therefore, it is assumed that the explosive events are caused by magnetic recomnection which occurs in a bursty manner along the boundary separating the opposite polarity element.s. (Dere et. al., 1991).

In this report, we assume that new magnetic flux typically emerges at the supergranular network cell centers as small bipoles. Then these fields are separated and convected with the typical velocity of intranetwork elements to the network boundaries where they 
cancel with fields of the opposite polarity. We have investigated the temporal evolution of localized recomnection process between supergranular cell field and emerging intranetwork flux using a compressible MHD code developed by Hu (1989) and Jin and Ip (1991). The numerical results show that the cancellation of magnetic flux and plasma accelerated by the nagnetic force in the reconnection process.

\section{MODEL CAC:ULATION}

The basic approach of our numerical calculation is based on the idea of asymmetric driven reconnection. That is, on left side of the simulation box, continuous inflow are imposed. Also, the electrical resistivity $\left(\eta_{m}\right)$ is the taken to be uniform everywhere. The $2 \mathrm{D}$ computation is excuted on the simulation box which extends from $-L_{x}=-l_{0}$ to $L_{s}=I_{0}$ and from 0 to $L_{z}=3 l_{0}$ in the $\mathrm{x}$ and $\mathrm{z}$ directions, respectively. In the following, both $\mathrm{x}$ and $\mathrm{z}$ are normalized by $l_{0}$. The area is divided into $44 \times 30$ grid points. Also note that the $x$ axis is normal to the current sheel while the $z$ axis is aligned with it. The introduction of a magnetic flux function which related to the magnetic field by $B=y \times \nabla A$ converts the equation of magnetic induction into a scalar equation.

We assmmed that emerging magnetic flux are separated and convected with the velocity of intranetwork elements to the network boundaries. The magnetic flux function at tine $t=0$ is given by

$A= \begin{cases}B_{01} x & -1 \leq x \leq-w_{1} \\ \frac{2 w_{1} B_{01}}{\pi} \cos \frac{\pi x}{2 w_{1}}-w_{1} B_{01} & -w_{1}<x \leq 0 \\ \frac{2 w_{2} B_{02}}{\pi} \cos \frac{\pi x}{2 w_{2}}-w_{2} B_{02} & 0<x \leq w_{2} \\ -B_{02} x & w_{2} \leq x<1\end{cases}$

i.e., the initial manetic field is

$$
\begin{aligned}
& B_{1}= \begin{cases}-B_{01} & -1 \leq x \leq-w_{1} \\
B_{01} \sin \frac{\pi x}{2 w_{1}} & -w_{1}<x \leq 0\end{cases} \\
& B_{2}= \begin{cases}B_{02} \sin \frac{\pi x}{2 w_{2}} & 0<x \leq w_{2} \\
B_{02} & w_{2} \leq x \leq 1\end{cases}
\end{aligned}
$$

where $B_{1}, w_{1}$ is the magnetic field and the current sheet lialf width on the side of emerging int.ranetwork: $B_{2}, w_{2}$ is the magnetic field and the current sheet half width on the side of network field. The initial magnetic field configuration is assumed to be in a mechanical equilibrium state, the total pressure remains constant, hence, the normalized plasma density are expressed respectively as $\rho_{1}=1+1 / \beta_{1}-B_{1}^{2} / \beta_{1}$ and $\rho_{2}=\rho_{02}+\left(1-B_{1}^{2}\right) Q^{2} / \beta_{1}$. Here $Q=B_{01} / B_{02}$ and $\beta_{1}=P_{01} /\left(B_{01}^{2} / 2\right)$ is the ratio of plasma pressure and magnetic pressure on the left side of the current sheet at time $=0$. The paranents on the emerging intranct.work side and on the network side are presscribed by the subscript 1 and 2 respectively. Note that in the present study, we set, $A_{0}=B_{01}$ (the initial magnetic flux function at $x=-1), \rho_{0}=\rho_{01}$. 
At left boundary $\left(x=-L_{x}=-1\right)$, we have $Y_{z}=0, T=1, \rho=1, \frac{\partial^{2} A}{\partial x \partial t}=0$, (i.e., $\left.\frac{\partial B_{x}}{\partial t}=0\right)$ and

$V_{z}= \begin{cases}M P_{A} V_{A} & 0 \leq z \leq 1 \\ M I_{A} V_{A}[\sin (1.5-z) \pi] & 1<z<1.5 \\ 0 & 1.5 \leq z \leq 3\end{cases}$

Along right boundary $\left(x=L_{x}=1\right)$, we set. $l_{x}=l_{z}=0, \mathrm{~T}=1, \rho=\rho_{02}, A=A_{02}$. The boundary condition at $z=L_{z}$ (top boundary) follows that $V_{r}=0$. Furthermore, $V_{z}, p$ and $\mathrm{T}$ are determined by the linear extrapolation method and $\frac{\partial^{2} A}{\partial z^{2}}=0$ (i.e., $\frac{\partial B_{c}}{\partial z}=0$ ). Along the bottom boundary $(z=0), V_{2}, \rho, T, A$ are guen by the same method as top boundary, however, $I_{x}$ is specified by the linear extrapolation method for left half part of bottom boundary $(x=-1 \ldots 0)$ and $V_{x}=0$ is set. for right half part of the bottom boundary $(x=0 \ldots-1)$. The full viscous MHD equation are solved using a multiple-step inplicit. scheme (Hu, 1989). Nole that the grid point spacing in $x$ direction is non-equidistant to allow adequate spatial resolution of current sheet. More details of this compressible MHD code which was used to study magnetic merging processes can be found in Jin and Ip (199I) and $I p$ and Jin (1991).

\section{SIMULATION RESULTS:}

In order to emphasize the reconfiguration of magnetic field topologies in the reconnection region, only the date for $|\boldsymbol{x}| \leq \mathbf{0 . 6}$ are used in the figure plotlings. In the present study we take $Q=B_{02} / B_{01}=1.5, B_{02}=18.62 G, l_{0}=1500 \mathrm{~km}, w_{1}=150 \mathrm{~km}, w_{2}=w_{1} / Q, \beta_{1}=$ $1.475, \beta_{2}=0.1, \rho_{01}=32.78 \times 10^{10} \mathrm{~cm}^{-3}, \rho_{02}=5 \times 10^{10} \mathrm{~cm}^{-3}, T_{0}=10^{5} \mathrm{~K}, R_{m}=$ 1500, $M_{A}=0.15$. The reconnection configuration between emerging intranetwork flux and the network field is shown in Fig.1. As shown in Fig.1 the emerging magnetic flux are driven by the incoming flow imposed at left boundary $\left(x=-L_{x}\right)$ then reconnected near the bottom boundary. The reconnection field lines(i.e. the contours of $A$ ) are convected upward throgh the boundary at $z=L_{i}$ which cause the manetic field lines to become more spare. This result is qualitatively in agreement wilh the cancellation of magnetic flux observed in the explosive events. Also, it can be seen from Fig.l that the foot points of magntic field lines are slowly moved toward the right side since the continuous action of the inconing flow imposed at left boundary. For the maximum speeds $V_{M}$ of the plasma upward jets from the reconnection region the values of our model calculalions are as follows: $0.303 V_{A 1}\left(\right.$ time $\left.=4.55 t_{A 1}\right), 0.338 V_{A 1}\left(\right.$ t.ine $\left.=0.505 t_{A 1}\right), 0.369 V_{A 1}$ $\left(\right.$ time $\left.=0.556 t_{A 1}\right), 0.405 V_{A 1}\left(\right.$ time $\left.=0.606 t_{A 1}\right)$; i.e. the plasma flows are accelerated in the recomnection process.

\section{DISCUSSION}

Making use of a 2D compressible MHD code, we have explored the reconnection process between emerging intranetwork flux and network field. The accelerated plasma and the cancellation of magnetic flux which might provide a link to the observed features associated with the explosive events are shown in the numerical results. The maximum ontflow speed of the plasma ejecting from the recomnection region is on the order of $0.4 V_{A 1}$ (ahont $20 \mathrm{~km} / \mathrm{s}$ ) which is substantially below the velocity of the bulk plasma in the explosive events. That is the reason why the small initial magnetic strengths and the 
considerable large $\beta$ value are set in our present study. In this reprot, we only present. the prelinminary results from a numerical simulation. Further examination of other cases with considerahly higher magnetic field strengths than in present study will be made. The high-velocity plasma outflow which is accelerated by the magnetic force $J \times B$ will be shown in the further study.
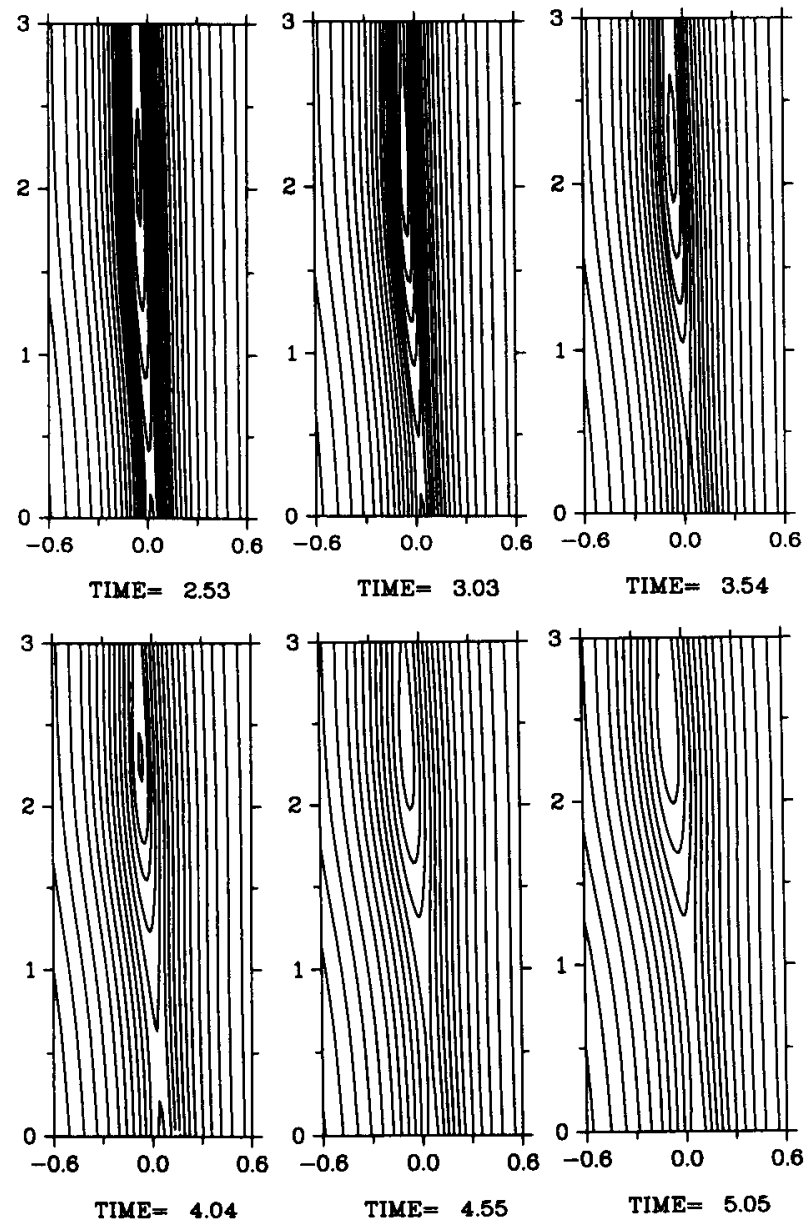

Figure 1 The time variations of the magnetic field configurations

\section{REFERENCES}

Dere, K.P., J.-D.F. Bartoe, G.E. Brueckner, et al., 1991, J. Geophys. Res., 96, 9399.

Hu, Y-Q., 1989, J.Comput. Phys., 84, 441.

Ip W.-H. and S.-P.Jin, 1991, Geophys. Res. Lett.. 18,1497.

Jin S.-P. and W.-H. Ip, 1991. Phys. Fluids, $3,1927$. 